\title{
CARACTERIZAÇÃO DA VOZ DO INDIVÍDUO PORTADOR DE OBESIDADE MÓRBIDA
}

\author{
Morbid obesity and it's relationship to voice alterations
}

\author{
Maria Gabriela Bernardo da CUNHA, Gustavo Haruo PASSEROTTI, Raimar WEBER, Bruno ZILBERSTEIN
}

ABCDDV/646

Cunha MGB, Passerotti GH, Weber R, Zilberstein B. Caracterização da voz do indivíduo portador de obesidade mórbida. ABCD Arq Bras Cir Dig 2009;22(2):76-81

RESUMO - Racional - A obesidade é doença crônica e grave com crescimento alarmante em todo o mundo e de interesse na saúde pública. Existe alteração no comportamento vocal destes pacientes e ele é pouco estudado - Objetivo - Caracterizar prospectivamente a voz de indivíduos portadores de obesidade mórbida comparando-a com a de não obesos. Métodos - Estudo prospectivo de 45 indivíduos adultos voluntários, 14 homens e 31 mulheres com idade média de 43 anos variando de 20 a 63 anos. Todos apresentavam IMC acima de $35 \mathrm{Kg} / \mathrm{m} 2$ constituindo o grupo em estudo (GO) Para cada indivíduo do GO foi selecionado também um indivíduo adulto voluntário, proveniente da população geral do ambulatório de otorrinolaringologia com IMC abaixo de $30 \mathrm{Kg} / \mathrm{m} 2$, constituindo o grupo controle (GN). Os critérios de exclusão foram diagnóstico de lesões neoplásicas malignas em cabeça e pescoço ou operações prévias da região orofaríngea. Fooram submetidos a exame laringológico e à gravação da voz. Também submeteram-se à avaliação subjetiva perceptivo-auditiva da qualidade vocal, através da escala GIRBAS e a aspereza, o estrangulamento no final das. A análise acústica foi realizada objetivamente utilizando os softwares Vox Metria versão 4.0.47 e PRAAT, fornecendo a frequência fundamenta, jitter, shimmer, ruído e tempo máximo fonatório. Resultados - Observou-se que os grupos foram homogêneos em relação ao sexo, idade, tabagismo e altura, mas significativamente diferentes em relação ao peso e IMC $(P<0,001)$. Os achados laringoscópicos em ambos os grupos mostraram que $26,7 \%$ dos obesos tinham laringoscopia alterada contra $13,3 \%$ dos não obesos. Sinais laríngeos sugestivos de RLF foram significativos para o GO $(P<0,042)$. A análise perceptivo-auditiva revelou que o grau da disfonia, a instabilidade, a rouquidão, a soprosidade, a astenia, a tensão e a aspereza tiveram alterações altamente significantes para o GO ( $P=0,001$ ou 002$)$. O estrangulamento ao final das emissões, bem como a ressonância da produção vocal foi significante $(P=0,001)$ comprometendo o GO. Na análise acústica notou-se que o jitter, shimmer, ruído e o tempo máximo fonatório apresentaram significância alta comprometendo o GO. Conclusão - A voz do indivíduo portador de obesidade mórbida apresenta alterações significantes quanto às características vocais em relação aos indivíduos não obesos, caracterizando a voz do obeso como rouca, soprosa, instável, estrangulada e com tempos máximos fonatórios reduzidos.

DESCRITORES - Obesidade. Alteração da voz. Avaliação da voz.

\section{INTRODUÇÃO}

A voz é o produto do ar expirado pelos pulmões que se sonoriza nas pregas vocais modificado pelas cavidades de ressonância como faringe, cavidade oral e nasal e por estruturas como lábios, língua e palato.

A fonação é ato físico de produção do som por meio da interação das pregas vocais com a corrente de ar exalada. Os puffs de ar são liberados em frequência audível, ressoando nas cavidades supraglóticas. Os atributos da voz normal são: "pitch" (sensação subjetiva da frequência) adequado à idade e sexo; "loudness" (sensação subjetiva da intensidade) adequado ao ambiente; "qualidade agradável" e sem ruídos (relativo à ressonância e ruídos glóticos); "flexibilidade" com expressões de ênfase; "significado e sutilezas" (interação de todos os anteriores).

A disfonia é, por definição, um sintoma caracterizado como "dificuldade na emissão da voz com suas características naturais"

Trabalho realizado na Disciplina de Cirurgia do Aparelho Digestivo do Departamento de Gastroenterologia da Faculdade de Medicina da Universidade de São Paulo, SP, Brasil.

Endereço para correspondência: Bruno Zilberstein, e-mail: brunozilb@uol.com.br
Parte importante da abordagem fonoaudiológica é a avaliação perceptivo-auditiva da voz que pode ser de fundamental importância para o diagnóstico correto e tratamento mais adequado. A avaliação perceptivo-auditiva é a clássica da qualidade vocal, tradicional da rotina clínica ${ }^{5}$. Segundo Colton, et al. ${ }^{7}$, é apropriado fazer um julgamento perceptual da qualidade vocal, porém tais julgamentos devem ser feitos com cautela.

Na tentativa de se diminuir a subjetividade da avaliação perceptivo-auditiva e conseguir-se padronização, algumas escalas foram criadas. A japonesa (GIRBAS), é baseada nos trabalhos sobre rouquidão ${ }^{10,11}$. Em 1996, Dejonckere, et al. ${ }^{8}$ propuseram que se acrescentasse o fator I, de instabilidade. Esta escala GIRBAS é particularmente centrada em nível laríngeo e oferece avaliação rápida, compacta e confiável, tendo-se transformado na mais utilizada internacionalmente. Ela é método simples de avaliação do grau da disfonia $(\mathrm{G})$ pela identificação de quatro parâmetros isolados: rouquidão - irregularidade de vibração das pregas vocais (R-roughness); instabilidade - flutuação na qualidade vocal (I-instability); soprosidade - escape de ar transglótico não sonorizado (B-breathness); astenia - fraqueza vocal, perda de potência com harmônicos pouco definidos (A-asteny); 
e tensão - compressão das pregas vocais na linha média (Sstrain). São considerados os mais importantes na definição de voz disfônica.

Algumas características da emissão vocal auxiliam na qualificação da escala. Por exemplo, o estrangulamento ao final da emissão significa que a voz está tensa. A vibração da mucosa das pregas vocais é de pouca extensão. Isto pode estar associado ao tempo máximo de fonação reduzido em que o fluxo de ar vai diminuindo e o indivíduo faz certa força estrangulando, assim, o final da emissão.

Atualmente, dados objetivos da qualidade vocal têm sido determinados pela avaliação acústica computadorizada, onde se pode obter informações objetivas dos parâmetros vocais. Esses dados podem ser utilizados para a avaliação da eficácia terapêutica, seja esta cirúrgica, medicamentosa ou fonoterápica. Entretanto, por mais completa que seja a análise acústica realizada, os dados numéricos obtidos dificilmente são capazes de substituir por completo a avaliação perceptivo-auditiva do examinador e do próprio paciente.

São avaliados na análise acústica a frequência fundamental, jitter, shimmer, ruído, tempo máximo fonatório, harmônicos e formantes.

É sem dúvida de interesse analisar o comportamento destes parâmetros em diferentes afecções prevalentes na população, no sentido de caracterizá-los e eventualmente modificá-los.

Em particular a obesidade - afecção de alta prevalência -, é doença crônica e grave, com crescimento alarmante em todo o mundo e constituindo-se em problema de saúde pública atualmente.

Torna-se desta forma objeto de interesse o estudo do comportamento vocal destes pacientes, mormente em função das alterações fisiopatológicas associadas à obesidade que comprometem virtualmente todos os sistemas do organismo ${ }^{13}$. Uma dessas alterações é a disposição corporal de tecido adiposo excessivo com deposição anormal de gordura na úvula, palato mole, paredes laterais e posterior da faringe, e região posterior da língua, estruturas estas pertencentes à composição do trato vocal ${ }^{14}$.

Moura ${ }^{14}$ descreve em seus estudos que, devido ao acúmulo anormal de gordura nas vias aéreas superiores, existe aumento do espessamento das paredes laterais da faringe, do tamanho da língua e do comprimento da massa do palato mole. Aloé, et al. ${ }^{1}$ relatam que, devido à presença desse depósito de tecido adiposo em região rinofaringe, orofaringe e laringe, existe modificação de estreitamento geométrico das vias aéreas superiores - traquéia extratorácica, faringe, laringe e nariz. Essa alteração provoca redução no funcionamento e na sensibilidade de químio-receptores respiratórios, o que será compensado por hiperatividade da musculatura dilatadora faríngea.

Neste contexto observa-se que os pacientes portadores de obesidade mórbida podem apresentar alterações próprias da voz.

A obesidade, hipertensão arterial e refluxo gastroesofágico são fatores associados à síndrome da apnéia e hipopnéia obstrutiva do sono (SAHOS). A obesidade é fator predisponente, particularmente pela adiposidade na parte superior do corpo, com aumento de tecido mole da faringe ou de tecido linfóide.

Apesar dos fortes indícios de que os indivíduos com obesidade mórbida apresentem característica vocal diferente dos normais, não se encontra na literatura estudo que delineie e caracterize a voz da população portadora dessa condição.

O objetivo deste estudo é caracterizar prospectivamente a voz de indivíduos portadores de obesidade mórbida comparando-a com a de não obesos, através da análise acústica computadorizada e perceptivo-auditiva.

\section{MÉTODOS}

Foram estudados 45 indivíduos adultos voluntários, 14 homens e 31 mulheres com idade média de 43 anos variando de 20 a 63 anos. Todos apresentavam IMC acima de $35 \mathrm{Kg} / \mathrm{m}^{2}$ constituindo o grupo em estudo (GO). Todos eram provenientes do Ambulatório de Obesidade da Disciplina de Cirurgia do Aparelho Digestivo do Departamento de Gastroenterologia da Faculdade de Medicina da Universidade de São Paulo, SP, Brasil. Para cada indivíduo do GO foi selecionado também um indivíduo adulto voluntário, proveniente da população geral do ambulatório de otorrinolaringologia da mesma instituição, pareados por sexo, idade ( \pm 1 ano) e tabagismo, com IMC abaixo de $30 \mathrm{Kg}$ / $\mathrm{m}^{2}$, constituindo o grupo controle (GN)(Tabela 1).

Os critérios de exclusão foram diagnóstico de lesões neoplásicas malignas em cabeça e pescoço ou operações prévias da região orofaríngea.

Todos assinaram consentimento pós-esclarecido e o projeto de pesquisa foi aprovado na Comissão de Ética da instituição sob protocolo de pesquisa número 1283/05.

Inicialmente, os indivíduos responderam a questionário direcionado para identificação de hábitos e comorbidades. Em seguida foram submetidos a exame laringológico no ambulatório de otorrinolaringologia e à gravação da voz no setor de fonoaudiologia do ambulatório de otorrinolaringologia. Os exames foram realizados sempre pelo mesmo médico e os diagnósticos analisados também por outros dois médicos independentes, especialistas em laringologia e voz, sendo o resultado de consenso.

Após a realização do exame de laringe foi realizada a gravação da voz, cuja interpretação foi analisada por três fonoaudiólogas independentes, sendo resultado de consenso.

O exame da laringe foi realizado com endoscópio rígido da marca $S_{\text {torz }}{ }^{\circledR}$ com lente de 70 o e registrado em DVD. Era categorizado como normal (sem lesões benignas de pregas vocais) ou alterado (com lesões benignas de pregas vocais). Os indivíduos foram considerados com tendo sinais laríngeos sugestivos de refluxo laringofaríngeo quando apresentavam paquidermia, hiperemia e/ou edema interaritenoideo, e/ou pseudo-sulco nas pregas vocais ${ }^{12}$.

Para a gravação da voz foi pedido aos indivíduos que emitissem as vogais /a/ e /é/ prolongadas, de maneira natural e confortável, em intensidade e altura habituais, sem impostar a voz, e contagem de números de 1 a 20 . Todas essas emissões foram gravadas em microcomputador e microfone headset unidirecional da marca Plantronics colocado à distância de três centímetros da boca de cada 
indivíduo em cabine com tratamento acústico.

Os indivíduos foram submetidos à avaliação subjetiva perceptivo-auditiva da qualidade vocal, através da escala GIRBAS. Foram avaliadas também a aspereza - tensão e redução da massa vibrante (H-harsh), e o estrangulamento ou fry no final das emissões - encurtamento máximo das pregas vocais. A gradação da escala GIRBAS e do H é de zero (sem alteração) a 3 (alteração severa). Quanto ao estrangulamento ou fry foi categorizada como presente ou ausente.

A determinação da escala GIRBAS, e de todos os outros parâmetros da análise perceptivo-auditiva, foram realizadas em uma mesma ocasião por três fonoaudiólogas especialistas em voz com experiência na área por no mínimo cinco anos, as quais não tinham conhecimento a qual grupo o indivíduo pertencia. Quando havia discordância entre as avaliações, o caso era reavaliado até que se chegasse a um consenso.

A análise acústica foi realizada objetivamente através do mesmo microcomputador utilizando os softwares Vox Metria versão 4.0.47 e PRAAT. Eles forneceram para cada indivíduo os valores numéricos de frequência fundamental (F0), jitter, shimmer, ruído e tempo máximo fonatório.

Os valores de referência utilizados neste estudo para frequência fundamental foi a média de $113 \mathrm{~Hz}$ e $205 \mathrm{~Hz}$ para homens e mulheres, respectivamente ${ }^{2,3}$. Os com valores acima de $0,6 \%$ foram considerados com jitter alterado. Os acima de $6,5 \%$ com shimmer alterado. Os indivíduos com valores acima de 2,5 foram considerados com ruído alterado.

Valores de referência de tempo máximo fonatório em falantes da cidade de São Paulo foi em torno de 20 segundos para homens e 14 para mulheres. Valores menores que 10 segundos foram considerados não-normais com alta significância ${ }^{4}$.

Para a análise estatística, as medidas de F0 e tempo máximo fonatório foram comparadas entre os grupos através do teste não paramétrico de Wilcoxon. As proporções de alteração nos valores de jitter, shimmer e ruído, bem como as proporções de fry e estrangulamento, foram comparadas entre os grupos utilizando-se o teste do qui-quadrado e o exato de Fisher. As proporções dos escores de cada item da qualidade vocal (GIRBAS e H-aspereza) foram comparadas entre os grupos através do teste do qui-quadrado. Valores de $P$ menores que 0,05 foram considerados estatisticamente significantes. Os dados foram analisados com o software Statistical Package for Social Sciences (SPSS ${ }^{\circledR}$ for Windows 10.0, SPSS Inc., Chicago, IL).

\section{RESULTADOS}

Na Tabela 1 estão expressos os dados demográficos referentes aos 90 indivíduos estudados. Observou-se que os grupos foram homogêneos em relação ao sexo, idade, tabagismo e altura, mas significativamente diferentes em relação ao peso e IMC $(P<0,001)$.

Os achados à laringoscopia em ambos os grupos estão apresentados na Tabela 2. Observou-se que 26,7\% dos obesos tinham laringoscopia alterada contra $13,3 \%$ dos não obesos. Sinais laríngeos sugestivos de RLF foram significativos para o $\mathrm{GO}(P<0,042)$.
TABELA 1 - Dados demográficos dos indivíduos estudados

\begin{tabular}{|c|c|c|c|}
\hline & \multicolumn{2}{|c|}{ Grupo } & \multirow[b]{2}{*}{$P$} \\
\hline & $\begin{array}{c}\text { Obesos } \\
(n=45)\end{array}$ & $\begin{array}{l}\text { Controles } \\
(n=45)\end{array}$ & \\
\hline \multicolumn{4}{|l|}{ Sexo } \\
\hline Masculino & $14(31,1 \%)$ & $14(31,1 \%)$ & \multirow[t]{2}{*}{1,0} \\
\hline Feminino & $31(69,9 \%)$ & $31(69,9 \%)$ & \\
\hline Idade (anos) & $43,3 \pm 12,0$ & $43,4 \pm 12,0$ & 0,86 \\
\hline \multicolumn{4}{|l|}{ Tabagismo } \\
\hline Não & $41(91,1 \%)$ & $41(91,1 \%)$ & \multirow{3}{*}{1,0} \\
\hline$<10 \mathrm{cig} / \mathrm{dia}$ & $3(6,7 \%)$ & $3(6,7 \%)$ & \\
\hline$\geq 10 \mathrm{cig} / \mathrm{dia}$ & $1(2,2 \%)$ & $1(2,2 \%)$ & \\
\hline Peso (Kg) & $131,4 \pm 25,6$ & $68,7 \pm 12,3$ & $<0,001$ \\
\hline Altura (m) & $1,65 \pm 0,11$ & $1,66 \pm 0,09$ & 0,54 \\
\hline $\operatorname{IMC}\left(\mathrm{Kg} / \mathbf{m}^{2}\right)$ & $48,3 \pm 6,4$ & $24,9 \pm 3,4$ & \multirow{7}{*}{$<0,001$} \\
\hline$<20$ & $0(0,0 \%)$ & $3(6,7 \%)$ & \\
\hline $20-24,9$ & $0(0,0 \%)$ & $20(44,4 \%)$ & \\
\hline $25-29,9$ & $0(0,0 \%)$ & $22(48,9 \%)$ & \\
\hline $30-34,9$ & $0(0,0 \%)$ & $0(0,0 \%)$ & \\
\hline $35-39,9$ & $5(11,1 \%)$ & $0(0,0 \%)$ & \\
\hline$\geq 40$ & $40(88,9 \%)$ & $0(0,0 \%)$ & \\
\hline
\end{tabular}

Dados apresentados como média \pm desvio padrão ou n (\%)

TABELA 2 - Prevalência de alterações à laringoscopia nos indivíduos estudados

\begin{tabular}{lccc}
\hline & \multicolumn{3}{c}{ Grupo } \\
& $\begin{array}{c}\text { Obesos } \\
(\mathbf{n}=\mathbf{4 5})\end{array}$ & $\begin{array}{c}\text { Controles } \\
(\mathbf{n}=\mathbf{4 5})\end{array}$ & $\boldsymbol{P}$ \\
\hline Laringoscopia & $33(73,3 \%)$ & $39(86,7 \%)$ & 0,11 \\
Normal & $12(26,7 \%)$ & $6(13,3 \%)$ & \\
Alterada & 2 & 1 & \\
Cisto de prega vocal & 1 & 0 & \\
AEM não especificada & 3 & 1 & \\
Cordite & 2 & 0 & \\
Espessamento nodular & 2 & 1 & \\
Granuloma de processo vocal & 1 & 0 & \\
Hipertrofia de banda & 1 & 0 & \\
Ventricular & 1 & 1 & \\
Úlceras de pênfigo vulgar & 1 & 1 & \\
Sulco de prega vocal & 0 & $10(22,2 \%)$ & $<0,042$ \\
Pólipo de prega vocal & 0 & &
\end{tabular}

Os resultados da análise perceptivo-auditiva (GIRBAS e aspereza) estão apresentados na Tabela 3. Notou-se que o grau da disfonia, a instabilidade, a rouquidão, a soprosidade, a astenia, a tensão e a aspereza tiveram alterações altamente significantes para o $\mathrm{GO}(P=0,001$ ou 002$)$.

Os resultados do vocal fry ou estrangulamento ao final das emissões, bem como a ressonância da produção vocal estão apresentados na Tabela 4. Observou-se que houve significância quanto ao estrangulamento $(P=0,001)$ comprometendo o GO.

Os resultados da análise acústica (Fo - frequência fundamental, jitter, shimmer, ruído, frequência máxima de harmônicos e TMF - tempo máximo fonatório) estão apresentados na Tabela 5. Notou-se que o jitter, shimmer, ruído e o tempo máximo fonatório apresentaram significância alta comprometendo o GO. 
TABELA 3 - Análise perceptivo-auditiva dos indivíduos estudados

\begin{tabular}{|c|c|c|c|c|c|c|c|c|c|}
\hline & \multicolumn{8}{|c|}{ Grupo } & \multirow{3}{*}{$\boldsymbol{P}$} \\
\hline & \multicolumn{4}{|c|}{ Obesos $(n=45)$} & \multicolumn{4}{|c|}{ Controles $(n=45)$} & \\
\hline & 0 & 1 & 2 & 3 & 0 & 1 & 2 & 3 & \\
\hline G & $\begin{array}{c}15 \\
(33,3 \%)\end{array}$ & $\begin{array}{c}28 \\
(62,2 \%)\end{array}$ & $\begin{array}{c}2 \\
(4,4 \%)\end{array}$ & $\begin{array}{c}0 \\
(0,0 \%)\end{array}$ & $\begin{array}{c}33 \\
73,3 \%)\end{array}$ & $\begin{array}{c}12 \\
(26,7 \%)\end{array}$ & $\begin{array}{c}0 \\
(0,0 \%)\end{array}$ & $\begin{array}{c}0 \\
(0,0 \%)\end{array}$ & 0,001 \\
\hline I & $\begin{array}{c}25 \\
(55,6 \%)\end{array}$ & $\begin{array}{c}19 \\
(42,2 \%)\end{array}$ & $\begin{array}{c}1 \\
(2,2 \%)\end{array}$ & $\begin{array}{c}0 \\
(0,0 \%)\end{array}$ & $\begin{array}{c}41 \\
(91,1 \%)\end{array}$ & $\begin{array}{c}4 \\
(8,9 \%)\end{array}$ & $\begin{array}{c}0 \\
(0,0 \%)\end{array}$ & $\begin{array}{c}0 \\
(0,0 \%)\end{array}$ & 0,001 \\
\hline $\mathbf{R}$ & $\begin{array}{c}17 \\
(37,8 \%)\end{array}$ & $\begin{array}{c}26 \\
(57,8 \%)\end{array}$ & $\begin{array}{c}2 \\
(4,4 \%)\end{array}$ & $\begin{array}{c}0 \\
(0,0 \%)\end{array}$ & $\begin{array}{c}33 \\
(73,3 \%)\end{array}$ & $\begin{array}{c}12 \\
(26,7 \%)\end{array}$ & $\begin{array}{c}0 \\
(0,0 \%)\end{array}$ & $\begin{array}{c}0 \\
(0,0 \%)\end{array}$ & 0,002 \\
\hline B & $\begin{array}{c}33 \\
(73,3 \%)\end{array}$ & $\begin{array}{c}12 \\
(26,7 \%)\end{array}$ & $\begin{array}{c}0 \\
(0,0 \%)\end{array}$ & $\begin{array}{c}0 \\
(0,0 \%)\end{array}$ & $\begin{array}{c}44 \\
(97,8 \%)\end{array}$ & $\begin{array}{c}1 \\
(2,2 \%)\end{array}$ & $\begin{array}{c}0 \\
(0,0 \%)\end{array}$ & $\begin{array}{c}0 \\
(0,0 \%)\end{array}$ & 0,001 \\
\hline $\mathbf{A}$ & $\begin{array}{c}45 \\
(100 \%)\end{array}$ & $\begin{array}{c}0 \\
(0,0 \%)\end{array}$ & $\begin{array}{c}0 \\
(0,0 \%)\end{array}$ & $\begin{array}{c}0 \\
(0,0 \%)\end{array}$ & $\begin{array}{c}45 \\
(100 \%)\end{array}$ & $\begin{array}{c}0 \\
(0,0 \%)\end{array}$ & $\begin{array}{c}0 \\
(0,0 \%)\end{array}$ & $\begin{array}{c}0 \\
(0,0 \%)\end{array}$ & 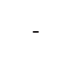 \\
\hline $\mathbf{S}$ & $\begin{array}{c}45 \\
(100 \%)\end{array}$ & $\begin{array}{c}0 \\
(0,0 \%)\end{array}$ & $\begin{array}{c}0 \\
(0,0 \%)\end{array}$ & $\begin{array}{c}0 \\
(0,0 \%)\end{array}$ & $\begin{array}{c}45 \\
(100 \%)\end{array}$ & $\begin{array}{c}0 \\
(0,0 \%)\end{array}$ & $\begin{array}{c}0 \\
(0,0 \%)\end{array}$ & $\begin{array}{c}0 \\
(0,0 \%)\end{array}$ & - \\
\hline H & $\begin{array}{c}44 \\
(97,8 \%) \\
\end{array}$ & $\begin{array}{c}1 \\
(2,2 \%)\end{array}$ & $\begin{array}{c}0 \\
(0,0 \%) \\
\end{array}$ & $\begin{array}{c}0 \\
(0,0 \%) \\
\end{array}$ & $\begin{array}{c}45 \\
(100 \%) \\
\end{array}$ & $\begin{array}{c}0 \\
(0,0 \%)\end{array}$ & $\begin{array}{c}0 \\
(0,0 \%)\end{array}$ & $\begin{array}{c}0 \\
(0,0 \%)\end{array}$ & 1,0 \\
\hline
\end{tabular}

Legenda: G (Grau da disfonia); I (Instabilidade); R (Rouquidão); B (Soprosidade); A (Astenia); S (Tensão); H (Aspereza)

TABELA 4 - Indivíduos estudados quanto ao vocal fry ou estrangulamento

\begin{tabular}{lccc}
\hline & \multicolumn{2}{c}{ Grupo } & \multirow{2}{*}{$\boldsymbol{P}$} \\
\cline { 2 - 3 } & Obesos $(\mathbf{n}=\mathbf{4 5})$ & Controles $(\mathbf{n}=\mathbf{4 5})$ & \\
\hline Vocal Fry & $8(17,8 \%)$ & $5(11,1 \%)$ & 0,37 \\
Estrangulamento & $10(22,2 \%)$ & $0(0,0 \%)$ & 0,001 \\
\hline
\end{tabular}

TABELA 5 - Análise acústica dos indivíduos estudados

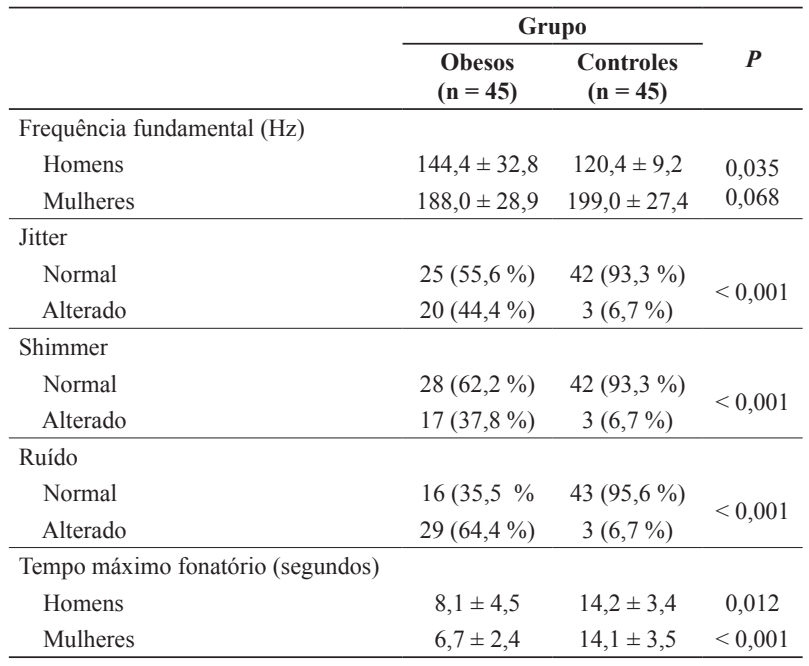

Dados apresentados como média \pm desvio padrão ou n (\%)

\section{DISCUSSÃO}

A obesidade mórbida como já é sabido é de alta prevalência no mundo ocidental e as suas consequências constituem verdadeiro problema de saúde pública.

As alterações da voz podem representar uma destas afecções consequentes, onde eventualmente os métodos terapêuticos possam interferir. Para tanto, é importante sem dúvida, caracterizar inicialmente a voz destes indivíduos. Esse foi o princípio que levou ao desenvolvimento deste estudo.

Para tanto, tornou-se muito importante a comparação prospectiva dos indivíduos obesos com voluntários normais. Desta forma procurou-se parear adequadamente os grupos, como pode-se observar na Tabela 1.

Também para melhor caracterizar os grupos e evitar falsos positivos foi realizado exame videolaringoscópico registrado em DVD e analisado em estudo duplo cego por outros profissionais da área sendo o resultado definido por consenso. Desta forma observou-se que as lesões laríngeas nos portadores de obesidade mórbida são de origem benigna ocorrendo nas pregas vocais em maior número absoluto inclusive do que nos indivíduos do GN. Entretanto estes valores não têm significância estatística $(P=0,11)$ demonstrando mais uma vez a homogeneidade da amostra.

Por outro lado tanto no GO quanto no GN foram evidenciados sinais sugestivos de RLF.

Este fato constituiu preocupação dos autores no sentido de analisar objetivamente a caracterização da voz do portador de obesidade mórbida, pois poderia ser aventado que este fenômeno induziria as alterações vocais encontradas e não ser consequente a todos os demais componentes anatômicos eventualmente alterados na obesidade mórbida. Desta forma foi realizado estudo estatístico comparativo no qual foram excluídos do GO todos aqueles que apresentassem sinais sugestivos de RLF e novamente pareados com os seus correspondentes do GN, também estes sem sinais sugestivos de RLF. Esta nova análise revelou que os indivíduos obesos mesmo sem sinais sugestivos de RLF, apresentam características vocais estatisticamente diferentes dos normais.

Como o indivíduo portador de obesidade mórbida apresenta alterações vocais independentemente da presença ou não de refluxo gastroesofágico, faz--se pensar que exista influência da obesidade na caracterização da voz.

Fica evidente pelos parâmetros da análise perceptivoauditiva e acústica da voz, que no GO vários parâmetros estão alterados.

Em relação à avaliação perceptivo-auditiva realizada através da escala GIRBAS, foi impactante a diferença observada entre o GO e o GN, onde praticamente todos os parâmetros estudados encontravam-se alterados. Observou-se claramente que a qualidade vocal do GO é significativamente alterada em relação aos indivíduos do GN. Assim, a voz dos indivíduos portadores de obesidade pode ser caracterizada como rouca (62\%), soprosa (27\%) e com instabilidade fonatória (44\%) (Tabela 3).

Esse achado concorda com os referentes à qualidade vocal do estudo de Bortolotti ${ }^{6}$, estudando mulheres obesas que encontraram qualidade vocal predominantemente rouca $(76 \%)$, soprosa (52\%) e crepitante (43\%).

Essas características vocais podem ser explicadas devido ao reduzido tempo fonatório apresentado pelos sujeitos estudados, o qual provocaria irregularidade de vibração das pregas vocais, por causa da reduzida pressão subglótica, levando assim à voz rouca.

A rouquidão é qualidade vocal do tipo ruidosa e indica irregularidade de vibração das pregas vocais ou aumento de massa $^{4,9}$. Essa irregularidade pode estar presente devido à reduzida pressão subglótica apresentada. Dessa forma, essa rouquidão pode, também, ser explicada por meio dos ruídos que são produzidos independentemente dos movimentos on- 
dulatórios da mucosa das pregas vocais, como, por exemplo, o ruído realizado pelos tecidos moles da orofaringe $\mathrm{e}^{1,14}$. Assim, ressalta-se a importância das características e integridade do trato vocal na produção sonora ${ }^{15}$ que, no caso dos indivíduos obesos, sofrem alteração pelo aumento de massa.

Quanto à qualidade vocal soprosa e instável, elas são facilmente explicadas em decorrência da reduzida pressão subglótica observada nos reduzidos tempos fonatórios. Essa soprosidade presente é indicativa de presença de fenda glótica durante a fonação.

Estes achados ainda não foram descritos na literatura por isso a necessidade de comparação destes parâmetros com os indivíduos normais.

Em relação ao estrangulamento, 10 indivíduos portadores de obesidade mórbida $(22 \%)$ apresentaram estrangulamento aos finais das emissões $(P<0,001)$. O estrangulamento da voz ao final da emissão significa que ela está tensa. A vibração da mucosa das pregas vocais é de pouca extensão. Isto pode estar associado ao tempo máximo de fonação reduzido em que o fluxo de ar vai diminuindo e o indivíduo faz certa força estrangulando assim, o final da emissão.

Quanto a analise acústica da voz, vários parâmetros encontraram-se alterados.

Em relação à frequência fundamental não se observou no grupo como um todo, diferença significante do $\mathrm{GO}$ em relação ao GN. Porém quando analisou-se apenas o sexo masculino esta diferença foi significante. Como este fenômeno é consequente à espessura das pregas vocais, pode-se inferir que nos indivíduos obesos do sexo masculino ela estaria maior em função da própria obesidade.

Deste modo, o jitter, o shimmer e o ruído foram significativamente diferentes no GO em relação ao GN. Deve-se salientar que esta alteração pode ser considerada anormal e portanto o padrão de voz alterado em relação a normalidade no GO.

Observou-se que 20 indivíduos obesos (44\%) e três do grupo controle (7\%) apresentaram jitter (sensação psicofísica da frequência) alterado. Este dado teve importância significativa $(P<0,001)$.

Behlau ${ }^{4}$ refere que, quando lesões afetam as pregas vocais, suas vibrações mostram aumento na aperiodicidade, o que é refletido em valores aumentados de jitter.

Este dado mostrou que, provavelmente, os indivíduos portadores de obesidade mórbida apresentem vozes mais aperiódicas em relação aos controles. Isto pode ser explicado pela prevalência eventualmente maior desses indivíduos em apresentarem RLF, o que causaria irritação na mucosa das pregas vocais tornando a vibração delas aperiódicas.

O shimmer, que por sua vez representa a sensação psicofísica da intensidade, apresentou valores alterados em 17 $(38 \%)$ e três ( $7 \%$ ) indivíduos portadores de obesidade e obesos respectivamente. Este dado mostra que, os primeiros, apresentam valores de shimmer significativamente mais alterados em relação ao $\mathrm{GN}(P<0,001)$.

Behlau ${ }^{5}$ refere que, como o jitter, o shimmer tende a ser geralmente elevado nos distúrbios laríngeos, apresentando também maiores valores nas frequências graves e de baixa intensidade.

Em relação ao ruído, 29 indivíduos obesos (64\%) o apresentavam em suas vozes, enquanto que em apenas três do GN
(7\%) ele foi encontrado $(P<0,001)$.

Já há referência que as vozes normais apresentam certa quantidade de ruído esperada Behlau ${ }^{5}$ e relacionada às perturbações da produção da voz, mas não em quantidades elevadas de ruído, como foi encontrado nos indivíduos portadores de obesidade mórbida. Esse dado pode estar relacionado às lesões benignas laríngeas encontradas ou à presença de RLF, as quais modificam os movimentos vibratórios da mucosa das pregas vocais ocasionando assim o ruído glótico.

Quanto ao tempo máximo fonatório que é representativo da eficiência glótica em relação à respiração, também foi significativamente diferente tanto nos homens quanto nas mulheres, indubitavelmente traduzindo a influência da pletora torácica do indivíduo obeso no fenômeno respiratório. Este fato é reforçado pela alteração detectada no estrangulamento que expressa a mesma dificuldade do indivíduo obeso de realizar movimentos respiratórios na mesma amplitude dos indivíduos normais.

Observou-se assim, que os indivíduos portadores de obesidade apresentam valores de tempo máximo fonatório muito reduzidos. Os homens do GN, apesar de apresentarem esse tempo abaixo dos valores encontrados por Behlau ${ }^{4}$, têm valores acima de 10 segundos, o que não foi encontrado nos indivíduos portadores de obesidade.

Yanagihara, et al. ${ }^{17}$, afirmam que o tempo máximo de fonação é alcançado por três fatores fisiológicos: capacidade do ar total disponível para a produção da voz, força expiratória e ajuste da laringe para o uso eficiente do ar, isto é, resistência glótica.

Moura ${ }^{14}$ comprova, por meio de ressonância magnética, que sujeitos obesos possuem a presença de depósitos gordurosos em face, região molar, tórax, língua, palato, faringe e região posterior e anterior da laringe. Também descreve em seus estudos que, devido ao acúmulo anormal de gordura nas vias aéreas superiores, existe aumento do espessamento das paredes laterais da faringe, do tamanho da língua e do comprimento da massa do palato mole.

Aloé, et al. ${ }^{1}$ relatam que, devido à presença desse depósito de tecido adiposo em região rinofaringe, orofaringe e laringe, existe modificação de estreitamento geométrico das vias aéreas superiores. Essa alteração provoca redução no funcionamento e na sensibilidade de químio-receptores respiratórios, o que será compensado por hiperatividade da musculatura dilatadora faríngea.

Assim, pode-se pensar que o valor reduzido dos tempo máximo fonatório dos indivíduos portadores de obesidade mórbida possa estar relacionado ao elevado peso destes indivíduos, levando a acúmulo de gordura na laringe, o que causa dificuldade nos ajustes das forças mioelásticas e aerodinâmicas da laringe, para boa produção vocal.

\section{CONCLUSÃO}

A voz do indivíduo portador de obesidade mórbida apresenta alterações significantes quanto às características vocais em relação aos indivíduos não obesos, caracterizando a voz do obeso como rouca, soprosa, instável, estrangulada e com tempos máximos fonatórios reduzidos. 
Cunha MGB, Passerotti GH, Weber R, Zilberstein B. Morbid obesity and it's relationship to voice alterations. ABCD Arq Bras Cir Dig 2009;22(2):76-81

ABSTRACT - Background-Obesity is a chronic disease with public health interest. Voice disturbance is common in this group. Aim - To compare the voice between the morbid obese patients with the non obese. Methods - Prospective trial with 45 adult volunteers with 43 years old median age with BMI $>35 \mathrm{Kg} / \mathrm{m}^{2}$ as study group and a control group in the same number but with BMI $<30 \mathrm{Kg} / \mathrm{m}^{2}$. Exclusion criteria were tumor in head and neck or previous operations in oropharynx. All had laryngoscopy and voice recording. They were submitted to GIRBAS score. Acoustic analysis was done based on Vox Metria software and PRAAT. Results - Laryngoscopy showed alterations in 26,7\% of the obese group and $13,3 \%$ in controls. The GIRBAS score was highly significant to the obese group. Acoustic alterations were also significant in the obese group. Conclusion - The patients with morbid obesity have significant alterations in voice caracteristics in comparison to the ones normal in weight.

HEADINGS -

\section{REFERÊNCIAS}

1. Aloé F, Pedroso A, Tavares S, Barbosa RC. Ronco e síndrome da apnéia obstrutiva do sono. Fono Atual 1997;1(1):34-9.

2. Behlau M, Madazio G, Feijó D, Pontes P. Avaliação de Voz. In: Voz: O Livro do Especialista - Volume 1. São Paulo, Revinter, 2001.

3. Behlau M, Pontes P. Avaliação e tratamento das disfonias. São Paulo: Lovise, 1995.

4. Behlau M, Tosi O, Pontes P. Determinação da freqüência fundamental e suas variações em altura (jitter) e intensidade (shimmer), para falantes do português brasileiro. Acta AWHO 1985;4(1):5.

5. Behlau M. Uma análise das vogais do português brasileiro falado em São Paulo: perceptual, espectrográfica de formantes e computadorizada da freqüência fundamental. São Paulo, 1984. Tese de Mestrado - Escola Paulista de Medicina.

6. Bortolotti P, Andrada e Silva MA. Caracterização da voz de um grupo de mulheres com obesidade mórbida acompanhadas no setor de cirurgia bariátrica da Irmandade Santa Casa de Misericórdia de São Paulo. Distúrbios da Comunicação, 2005;17(2):149-160.

7. Colton R, Casper J. Compreendendo os Problemas de Voz - Uma perspectiva fisiológica ao diagnóstico a ao tratamento. Porto Alegre, Artes Médicas, 1996.

8. Dejonckere P, Remacle M, Freznel E. Reliability and relevance of differentiated perceptual evaluation of pathological voice quality. In: Clemente, M.P. (Ed.) Voice Update. Amsterdam: Elsevier, 1996, pp321-4.
9. Gonçalves S, Nassif PAN, Milléo FQ, Ribas-Filho JM. Alterações na prega vocal em pacientes com refluxo laringofaríngeo expostos ou não ao tabagismo e abuso vocal. ABCD Arq Bras Cir Dig 2004; 17(1)

10. Hirano M. Psycho-acoustic evaluation of voice. In: Clinical Examination of Voice, New York, Springer Verlag, 1981.

11. Isshiki N, Yanagihara N, Morimoto M. Approach to the objective diagnosis of hoarseness. Folia Phoniatr (Basel). 1966;18(6):393-400.

12. Koufmann J. Gastroesophageal reflux and voice disorders. IN: Rubin JS, Sataloff RT, Korovin G, Gould WJ. Diagnosis and treatment of voice disorders. New York: Igaku-Shoin, 1995, pp 161-175.

13. Mancini MC. Obstáculos diagnósticos e desafios terapêuticos no paciente obeso. Arq Bras Endocrinol Metab 2001;45(6):1-44.

14. Moura SMGPT. Efeito agudo do CPAP nasal no controle ventilatório e nacapacidade de exercício de pacientes normocápnicos com síndrome da apnéia do sono obstrutiva [dissertação]. São Paulo: Universidade Estadual Paulista de Medicina; 1996

15. Sataloff RT. The professional voice: anatomy, function and general health. J Voice $1987 ; 1(1): 92-104$.

16. Tarneaud J. Traité pratique de phonologie et phoniatrie. Paris. Maloine, 1941

17. Yanagihara N, Von Leden M. Respiration and phonation. Folia Phoniatr 1967;19:153-66
Fonte de financiamento: não há Conflito de interesse: não há Recebido para publicação: 06/02/2009 Aceito para publicação: 03/03/2009 